\title{
Feuille de route pour promouvoir la relève dans la recherche clinique
}

\section{Katrin Crameri}

Dr, division Biomédecine, Office fédéral de la santé publique

La relève est insuffisante dans le domaine de la recherche clinique. Telles sont les conclusions d'un rapport élaboré conjointement par l'Académie suisse des sciences médicales (ASSM) et l'Office fédéral de la santé publique (OFSP), publié en 2014. Pour améliorer la situation, les parties prenantes ont élaboré une feuille de route dans le but de soutenir de façon ciblée et systématique les médecins intéressés par la recherche clinique à chacune des étapes de leur carrière.

En Suisse, la recherche clinique est longtemps restée dans l'ombre de la recherche fondamentale biomédicale. Ces dernières années, de nombreuses initiatives ont été prises pour renforcer la recherche clinique et sa qualité, intensifier la coordination nationale et le réseautage international, mais aussi créer le cadre nécessaire à une recherche clinique de qualité.

En 2014, l'ASSM et l'OFSP ont publié conjointement un rapport sur la relève dans la recherche clinique en Suisse [1]. Il ressort de ce document, élaboré dans le cadre du plan directeur de la Confédération concernant la recherche et la technologie biomédicales [2], que la relève n'a pas été suffisamment encouragée dans ce domaine.

L'OFSP a alors institué un groupe de travail jouissant du soutien des parties prenantes et des acteurs de la recherche clinique en Suisse. Cette task force réunissait notamment des représentants des facultés de médecine et des hôpitaux universitaires ainsi que les présidents de l'ASSM et de la Swiss Clinical Trial Organisation (SCTO). Elle était chargée de formuler des mesures ad hoc, de définir les responsabilités et d'établir un calendrier réaliste pour mettre en œuvre avec succès et de manière pérenne les recommandations présentées dans le rapport. C'est ainsi qu'elle a rédigé une feuille de route pour les années 2016 à 2021 [3], qui vise à promouvoir la relève dans la recherche clinique au moyen de cinq trains de mesures (ou work packages, ci-après WP). Le $W P 1$ vise à soutenir les programmes MD-PhD locaux dans le domaine de la recherche clinique. Le WP 2 prévoit la définition de normes minimales pour les compétences en recherche clinique afin de faciliter l'acquisition des compétences nécessaires aux médecins envisageant de s'orienter vers la recherche.
Le $W P 3$, pivot central de la feuille de route puisqu'il est lié à tous les autres $W P$, comprend la mise sur pied d'une école virtuelle, c'est-à-dire d'une structure faîtière coordonnant au niveau national les formations de base, postgrade et continue ainsi que des cours cadre supplémentaires. Cette structure permettra aux personnes intéressées d'acquérir de manière efficiente les qualifications nécessaires pour travailler dans le domaine de la recherche clinique et d'accéder facilement à toutes les informations utiles. Ses objectifs seront les suivants:

- fournir aux étudiants un soutien solide et durable pendant la phase de spécialisation (un soutien professionnel, administratif et financier, de même qu'un accompagnement relevant du mentorat);

- mettre au point une structure commune, de même qu'un programme/processus pré-organisé pour la formation:

- optimiser la compatibilité avec toutes les filières de spécialisation en médecine;

- éviter que la phase de spécialisation se prolonge inutilement;

- faciliter la mobilité des chercheurs à travers la Suisse.

Le WP 4 porte sur la création d'un programme de financement (pilote) sponsorisé par des fonds privés. Il sera ainsi possible d'allouer des bourses à de jeunes talents triés sur le volet. Le WP 5 concerne les facultés de médecine et les hôpitaux universitaires, chargés d'élaborer ensemble un concept pour assurer des conditions de travail et des perspectives de carrière attrayantes pour les jeunes chercheurs. Ce module est jugé hautement prioritaire, l'impact global de la feuille de route dépendant du succès de son implémentation. 
Les activités prévues se fondent sur les infrastructures et expertises existantes, ce qui renforcera l'efficacité et évitera les doublons. La mise en œuvre de la feuille de route incombe aux graduate schools locales proposant des filières MD-PhD, à la SCTO, au réseau des Clinical Trial Units, à l'ASSM, à l'OFSP et à unimedsuisse. De 2016 à 2018, les mesures vont être planifiées et réalisées de sorte qu'une structure cadre complète pour la formation et le soutien à la recherche clinique soit opérationnelle à partir du semestre d'automne 2018. Une phase pilote est prévue pendant trois ans; elle fera l'objet d'un suivi étroit qui se conclura par une évaluation.

La feuille de route vise à combler les lacunes constatées dans la relève en recherche clinique et à offrir aux chercheurs des possibilités de se former efficacement au plus haut niveau. Elle devrait donc inciter davantage de jeunes médecins à opter pour une carrière dans ce domaine. Si l'on veut que la recherche clinique axée sur le patient puisse continuer à se développer en maintenant son niveau d'excellence et que la population suisse puisse bénéficier rapidement des avancées scientifiques de chercheurs de haut vol, il est indispensable de disposer de professionnels dûment formés.

\section{Références}

1 OFSP/ASSM (2014): Relève pour la recherche clinique en Suisse. Rapport du groupe thématique. Berne. Disponible à l'adresse: http://www.bag.admin.ch/fr/masterplan

2 DFI/OFSP (2013): Mesures de la Confédération afin de renforcer la recherche et la technologie biomédicales. Berne. Disponible à l'adresse: http://www.bag.admin.ch/fr/masterplan

3 OFSP/parties prenantes (2016): Feuille de route 2016-2021 pour promouvoir la relève dans la recherche clinique. Berne. Disponible à l'adresse: http://www.bag.admin.ch/fr/masterplan 